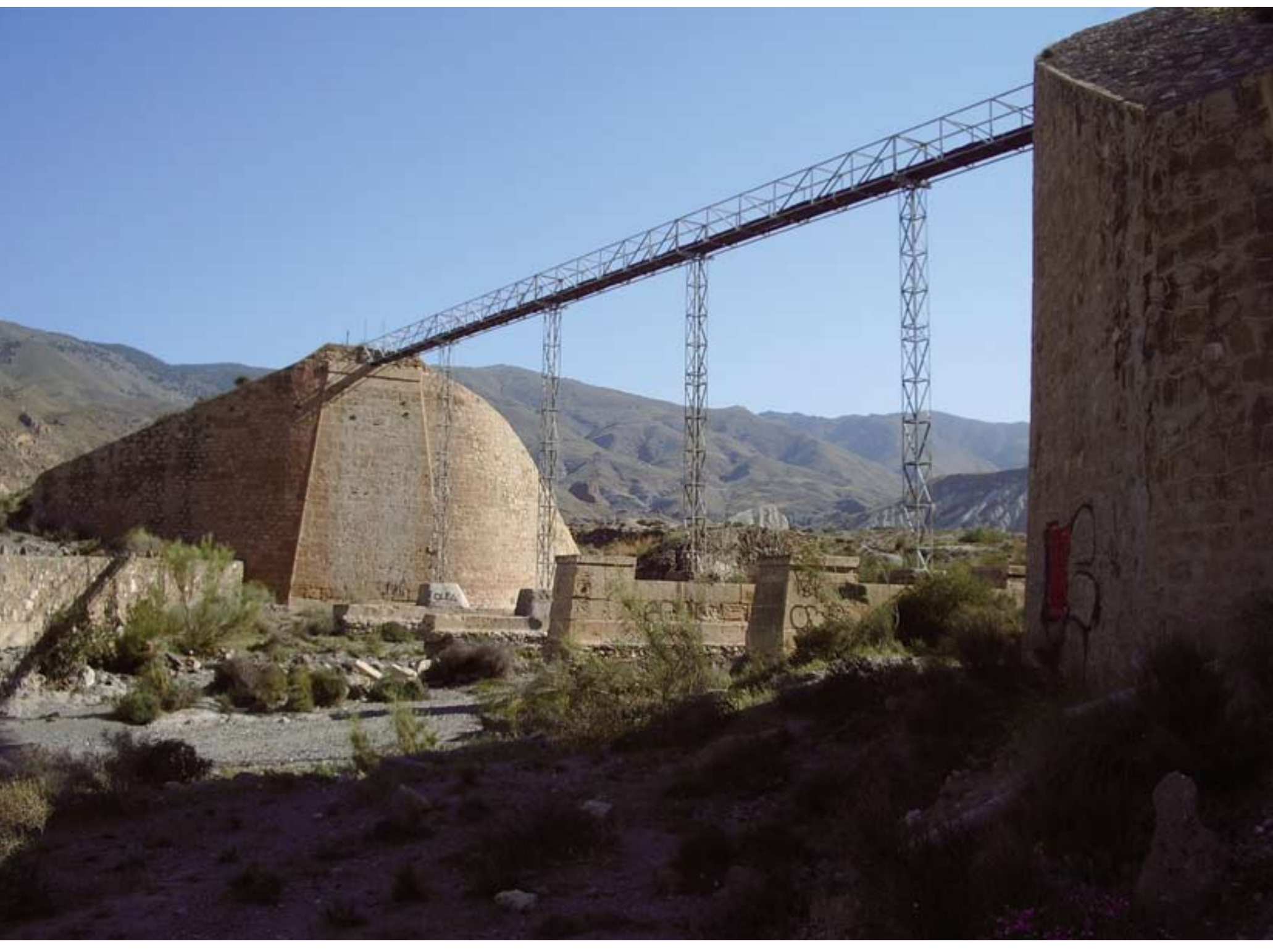

Puente de la rambla de Espinaza. Fuente: Fondo ASAFAL 


\section{Itinerario por el antiguo ferrocarril de Sierra Alhamilla a Almería: recuperación de un trazado para los ciudadanos}

Antonio Aguilera Cantón,

Asociación de Amigos del Ferrocarril de Almeria, ASAFAL

\section{ORIGEN DEL FERROCARRIL}

Concebido a finales del siglo XIX para transportar mineral de hierro desde varias explotaciones mineras de la parte occidental de Sierra Alhamilla hasta la playa de las Almadrabillas de Almería, fue el primer ferrocarril que llegó a la capital almeriense. Su existencia fue azarosa desde su misma gestación y tuvo una vida corta, sin embargo, nos ha quedado un legado patrimonial que deberíamos proteger del deterioro físico y del olvido.

Antes de "pisar" el terreno por donde circularon unas pequeñas locomotoras de vapor que arrastraban pesadamente los vagones cargados con mineral de hierro, debemos repasar brevemente la historia de este modesto ferrocarril minero, que se inicia con la puesta en marcha de la explotación de varios yacimientos de mineral de hierro en Sierra Alhamilla, impulsados por un empresario neoyorkino: William Dexter Marvell. Éste decide construir un ferrocarril que sacara la producción hasta el puerto de Almeria y aprovechar, al mismo tiempo, el trazado para comunicar los pueblos del bajo Andarax con los famosos baños medicinales de Sierra Alhamilla y la propia capital. En 1886 comienzan las obras y en 1888 está en servicio. Sin embargo, desde un principio el negocio fue ruinoso y las minas funcionaron irregularmente y no cumplieron con las expectativas depositadas en ellas; ni siquiera el transporte de viajeros aportaba lo suficiente para hacer rentable al ferrocarril. Ante esta situación, los acontecimientos se suceden y la propiedad de las minas y el propio ferrocarril se transfieren varias veces desde que The Marvell Iron Co Ltd. fuera la primera sociedad en el negocio en 1886, pasando por The Almeria and Alhamilla Railway Company Limited, The Gergal Railway and Mines Company Limited, The Alquife Mines and Railway Company Limited y The Chorrillo Almeria Railway Company Limited, respectivamente, hasta que, alrededor de 1919, las minas van agotándose o inundándose de agua al llegar al nivel freático. El 17 de noviembre de 1927 circuló el último tren y con ello propició el abandono y el expolio inmediato de todos los bienes.

Este ferrocarril minero tenía una longitud total entre El Chorrillo y el muelle de Almería de 15.859 m, con una pendiente me- dia de 22\%, siendo la sección más dura la parte alta junto a la sierra. Curiosamente se eligió un ancho de vía inusual: $914 \mathrm{~mm}$, equivalente a 3 pies ingleses, medida que en España solamente adoptaron los ferrocarriles de Mallorca y Sóller (Baleares) y el ferrocarril de Irún al Puente de Endarlaza (Guipúzcoa). A lo largo del recorrido existían cinco estaciones: Chorrillo, Alfaro-apeadero, Pechina, Huércal y Almería, de las que únicamente subsisten la primera y la tercera, aunque en un estado de ruina. A las estaciones de Chorrillo y Alfaro llegaban los cables aéreos con el mineral procedente de varias minas $y$, por tanto, eran los puntos de carga del ferrocarril.

El parque móvil que disponia la compañia para la explotación diaria era de tres pequeñas locomotoras de vapor alemanas tipo 030WT Jung que llevaban los nombres de Almeria, Pechina y Huércal, y que fueron desguazadas hacia 1946. El material remolcado lo componian unos 40 vagones para mineral de dos ejes con una carga media de $8 \mathrm{tm}$., además de otros vagones de varios tipos. También contaban con varios coches para viajeros de $2^{\text {a }}$ y $3^{\text {a }}$ clase de dos ejes y un coche break para los directivos; todos fueron desguazados. Habitualmente circulaba un tren diario cargado con mineral de hierro entre El Chorrillo y el muelle de Almería, descargándose en la playa de las Almadrabillas para su posterior embarque en barcazas hasta los buques fondeados en la rada. En cuanto a los trenes de viajeros, en los mejores tiempos del ferrocarril hubo cuatro o más trenes, hasta que la crisis redujo el servicio a dos semanales $y$, finalmente, uno sólo los domingos. Además de los trenes puros, también hubo trenes mixtos (viajeros y mercancias).

A pesar de que la mayoría de los elementos de este pequeño ferrocarril no existen, aunque muy deteriorados, aún podemos disfrutar todavía de algunos de sus restos, amenazados tanto por el paso del tiempo como por la especulación inmobiliaria y en medio de la desidia absoluta de las administraciones. $Y$ eso que una buena parte de la línea presenta cualidades excepcionales para su puesta en valor como vía verde, por lo favorable de su trazado, fácil acceso, su cercanía a núcleos poblados y la exótica belleza de algunos de los parajes que atraviesa. Un recorrido por 


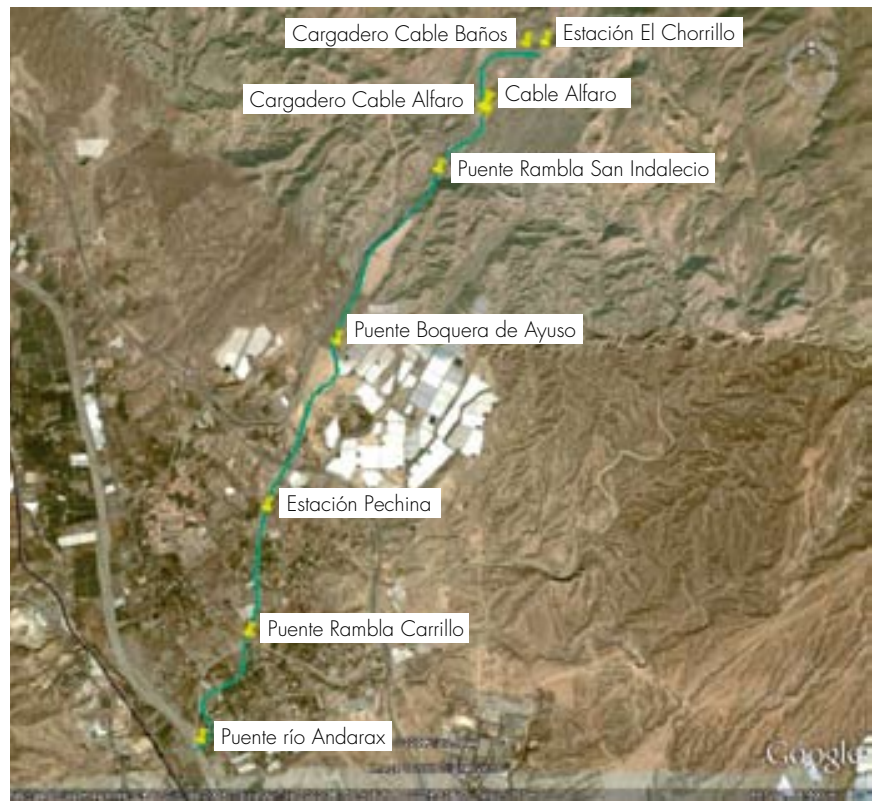

Itinerario por el antiguo ferrocarril de Sierra Alhamilla a Almería. Mapa: Mario López Martínez, a partir de Google Maps

sus vestigios nos pueden ayudar a comprender cuan importante es preservar nuestro patrimonio industrial para las presentes y futuras generaciones.

\section{RECORRIDO POR EL TRAZADO FERROVIARIO}

El trazado original ha desaparecido por completo desde la vieja estación de Pechina, aún en pie y perfectamente reconocible, hasta Almería. El desarrollo agrícola y urbano de los municipios por los que atravesaba el ferrocarril (Pechina, Huércal de Almeria y la propia capital) ha borrado cualquier vestigio reconocible de la traza o se ha reconvertido en una calzada asfaltada, como es el caso de la denominada carretera de Sierra Alhamilla en Almería. Sin embargo, milagrosamente, aún permanece casi intacta la parte alta o inicial de la línea que, sin lugar a dudas, es la más espectacular y objeto de nuestro interés.

El recorrido se inicia en la cabecera de la línea, en la falda de Sierra Alhamilla y en las proximidades de los baños termales del mismo nombre, en el paraje denominado El Chorrillo, a una altitud de $360 \mathrm{~m}$ aproximadamente. No es fácil reconocer lo que fue el kilómetro cero de la línea ferroviaria: estación, depósito de locomotoras, viviendas de ferroviarios y mineros, cantina, el cargadero de minerales y la propia traza de la vía. Todo está en estado de ruina y el lugar es escenario de batallas incruentas entre aficionados al paintball; a pesar de todo, son perfectamente reconocibles si nos apoyamos en un plano que reconstruye estas instalaciones (GÓMEZ MARTíNEZ; COVES NAVARRO, 2000: 114, vista aérea $\left.n^{\circ} 8\right)$.

Tras recorrer los distintos edificios que aún quedan en pie, no queda más que adentrarse en el trazado desde la propia esta- ción, sorteando previamente un pequeño pontón, que nos lleva hasta el cargadero del cable de El Chorrillo, del que tan solo queda el muro de mampostería paralelo a las vías y donde se aprecia la vía de culatón, que facilitaba las maniobras de los trenes cargados y vacios. En ese punto, tomamos la traza de la izquierda para salir poco a poco al Ilano por la explanación, perfectamente definida, hasta que se interrumpe bruscamente por culpa de los movimientos de tierras que hace algunos años se iniciaron con la intención, al parecer, de construir un invernadero agrícola o algo similar y que no llegó a culminarse. A pesar de ello, es fácil volver a la traza si tomamos como referencia las ruinas del segundo cargadero de mineral, el del cable Alfaro, para ir acercándonos por un terraplén cada vez más acusado que nos llevará hasta la primera gran obra de ingeniería de este ferrocarril minero: el espectacular puente de Espinaza, que salva la rambla del mismo nombre; con una longitud de $43 \mathrm{~m}$, conserva los estribos y cuatro pilastras de silleria de apoyo intermedio de la estructura metálica que formaba el puente, hoy desaparecida. En la actualidad, la explanación del ferrocarril ha sido aprovechada para canalizar las conducciones de agua y alcantarillado del núcleo de los Baños de Sierra Alhamilla, por lo que se han construido unos soportes metálicos, tanto en este puente como en el siguiente. No es posible transitarlo y, por ello, hay que retroceder unos metros para bajar por el terraplén de la vía y llegar a la rambla, desde donde se contempla una vista majestuosa de esta obra civil. Llegados a este punto, resulta imposible seguir sin mencionar que en 1961 sirvió de decorado cinematográfico para el primer western filmado en tierras almerienses, Tierra brutal, donde tiene lugar una de las muchas escenas rodadas en estos parajes ferroviarios. Pero esto no quedó en una simple anécdota, sino que todo el entorno de la cabecera de la estación de El Chorrillo ha servido en muchas ocasiones como escenario natural y lugar donde montar distintos decorados de diversas películas de cine y televisión, así como anuncios publicitarios. Sirvan de ejemplo títulos como El Evangelio de Juan, Las pistolas no discuten, El Halcón y la presa, Tú perdonas... yo no, Las maletas de Tulse Luper o Hermanos.

A partir del paso de este cauce, el trazado discurre en trinchera por la margen izquierda de la denominada rambla de San Indalecio, a una altura respetable de su lecho, por lo que no se puede acceder hasta unos metros aguas abajo, salvo que queramos subir con cierto riesgo. Una vez retomada la explanación, es muy interesante volver hacia el puente para contemplar las hermosas vistas de toda Sierra Alhamilla y tener una idea del desnivel que tuvo que afrontar este ferrocarril. También, es muy probable toparse con lagartos, culebras de respetable tamaño y distintas aves que sobrevuelan la traza.

Si se hace el recorrido en bicicleta hay que tener precaución con el terreno irregular y los pequeños derrumbes o corrimientos de tierra, fruto de la erosión y el paso de los años. Además, al ser descendente, se va cogiendo velocidad sin querer. Esta zona es quizás la más "ferroviaria" del trazado al discurrir en trinchera 


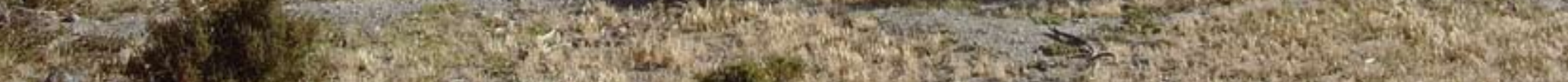
X.t.

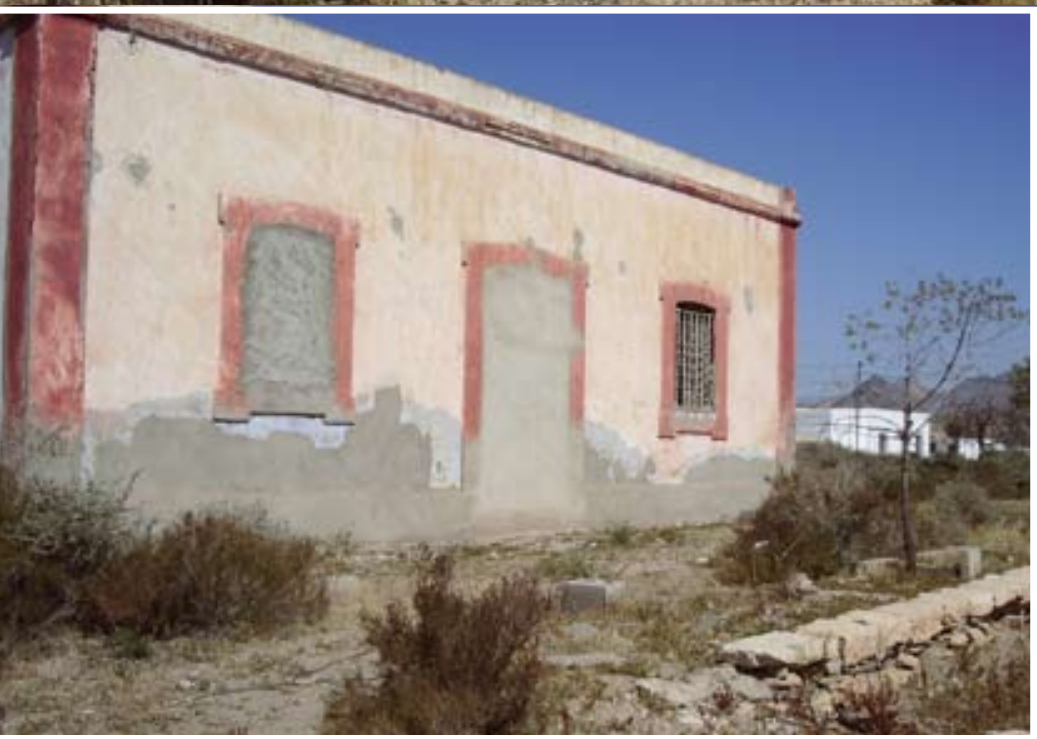

Viviendas de obreros en El Chorrillo. Fuente: Fondo ASAFAL

Estación de Pechina vista desde el andén. Fuente: Fondo ASAFAL

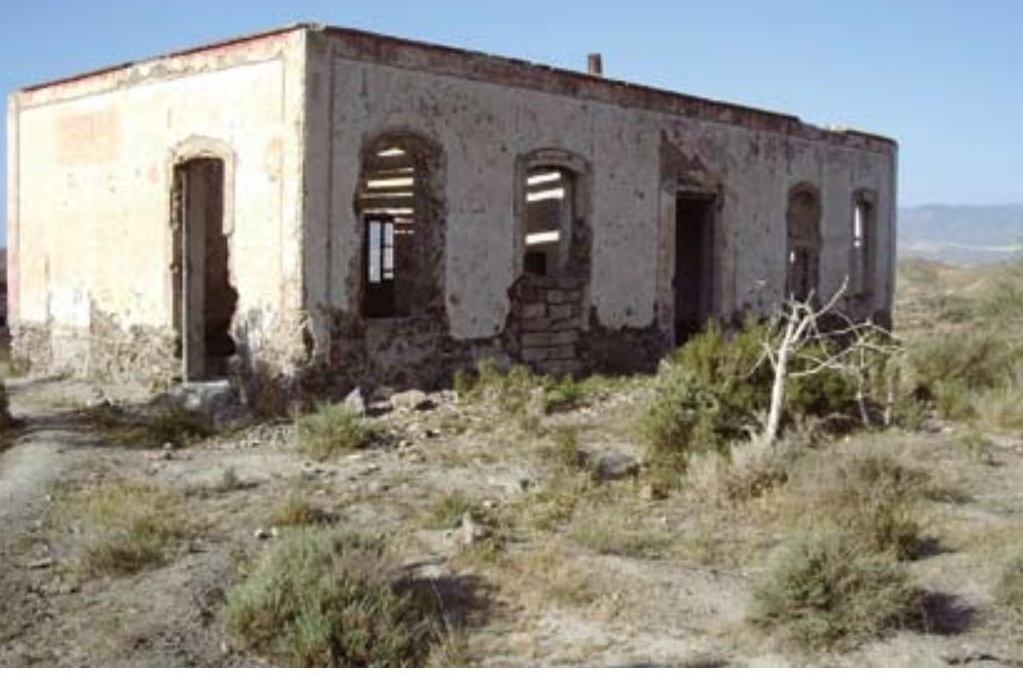

Estado actual del edificio de viajeros de El Chorrillo. Fuente: Fondo ASAFAL 


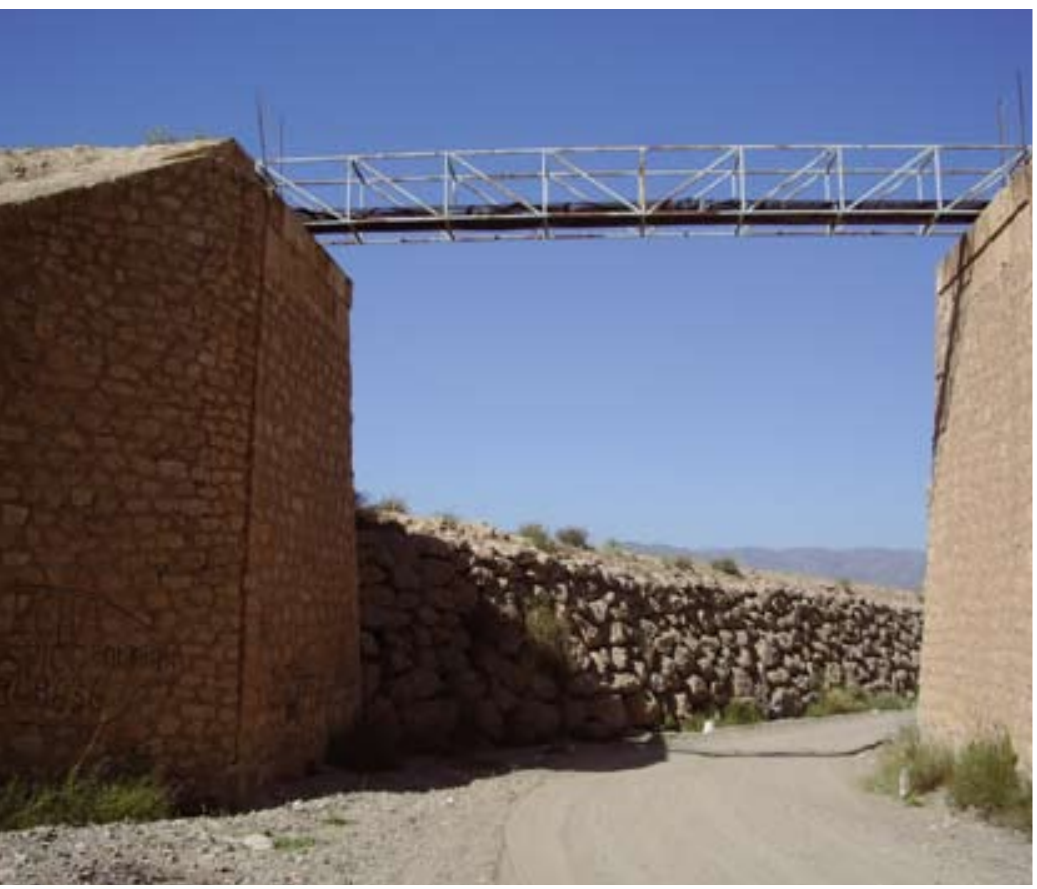

Puente de la rambla de Ayuso. Fuente: Fondo ASAFAL

antes de llegar al siguiente puente que atraviesa otra rambla, la denominada Ayuso. En este punto, se divisa perfectamente la costa y, como un castillo en un altozano, el edificio del hospital de Torrecárdenas de Almería. Al igual que el anterior puente, aquí se precisa desandar lo andado unos metros para bajar al lecho seco y pasar al otro lado, aunque, en este caso, ya entramos en una zona agrícola con invernaderos y la traza es aprovechada como camino rural asfaltado. Los sólidos estribos del puente soportan la leve y fea estructura metálica que albergan las conducciones de la red de agua y alcantarillado que termina en la próxima depuradora. El único tablero metálico que soportaba el paso de los trenes tenía una longitud de 10,60 m y sólo permanecen los estribos de mampostería.

Pasar al otro extremo del puente requiere dar un gran rodeo por un camino asfaltado, porque toda la margen izquierda de la rambla ha sido reforzada con grandes bloques de piedra para consolidar el terreno e impide una subida directa. En cualquier caso, aquí se puede dar por finalizado el tramo más "virgen" de este antiguo ferrocarril, porque, como ya se indicaba anteriormente, desde este punto la explanación se pierde casi por completo. Esa frontera, entre la parte alta y la baja estaría en el parque periurbano ubicado junto a una depuradora de aguas residuales que, posiblemente, destruyera el trazado original al realizar el desmonte y movimientos de tierras previo a su construcción. A partir del parque se puede continuar por la traza asfaltada para llegar a la estación de Pechina, o lo que queda de ella: un edificio de una sola planta que se asemeja más a una vivienda que a lo que entendemos por una estación de ferrocarril. Los restos del pequeño andén y un respiradero de una mina de agua (qanat árabe), protegida con un mallazo metálico debajo

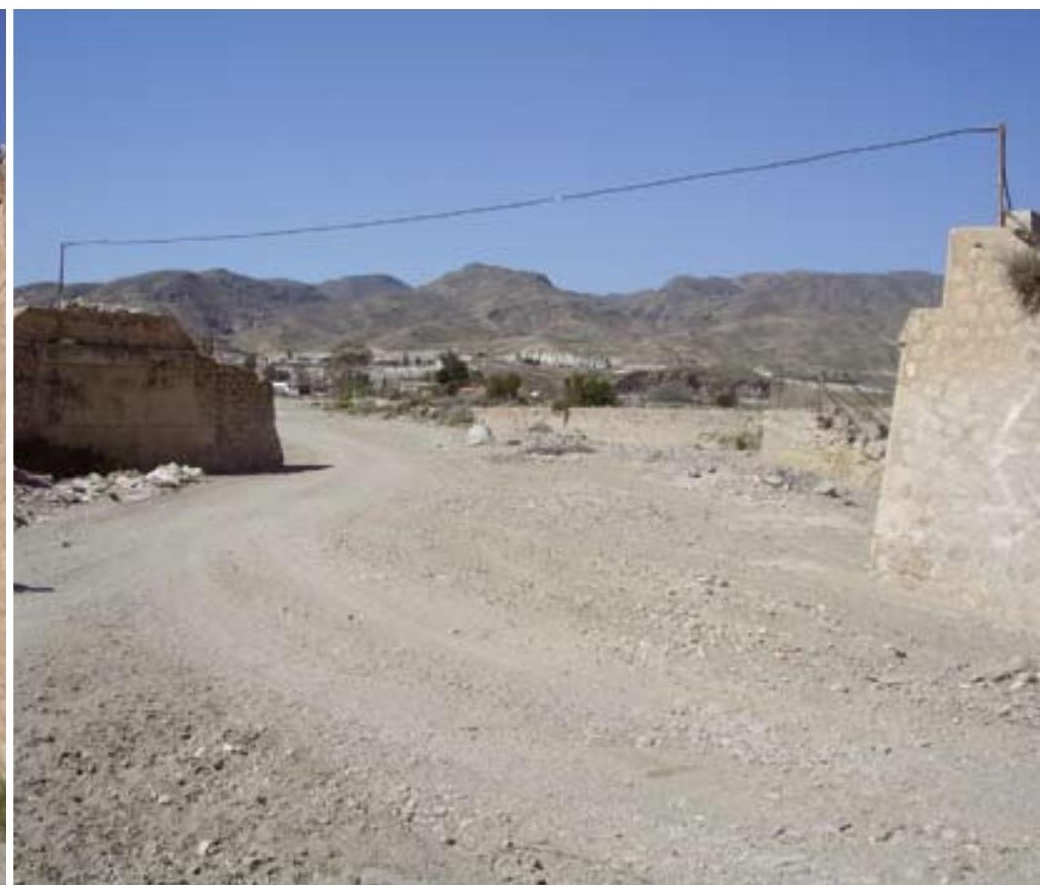

Puente de la rambla Carrillo. La pilastra de apoyo intermedio ha desaparecido. Fuente: Fondo ASAFAL

del cual todavía permanecen unos carriles, posiblemente de la propia línea, nos invitan a descansar.

Para continuar, es preciso seguir en línea recta por un camino de tierra e imaginar que, entre lo que hoy son bancales medio abandonados, iria el trazado ferroviario. Este camino va paralelo al mismo porque, entre algunos cortijos, se divisan los estribos del puente de la rambla de Parra, similar a la de Ayuso, y que nos da una idea de la ubicación de la explanación original.

El camino termina en la rambla de Carrillo, donde podemos observar un nuevo puente para salvar el cauce. Con una longitud de 21,40 m, estaba dividido en dos tramos metálicos con una pilastra central de sillería de apoyo, hoy desaparecida, subsistiendo solamente los estribos.

Ya resulta imposible volver al trazado ferroviario, porque, sencillamente, no existe, por lo que hay que descender por la misma rambla de Carrillo para desembocar en el cauce del río Andarax $y$, pocos metros aguas abajo, toparse con lo que en su día fue un magnífico puente metálico de $90 \mathrm{~m}$ del que sólo quedan los basamentos de piedra de cantería y los estribos de ambos lados. Este fue el punto más conflictivo que tuvieron que sortear los ingenieros que construyeron el ferrocarril minero y, por eso, optaron por un puente metálico dividido en nueve tramos de 7,70 y dos de 9,70 m, apoyados sobre columnas tipo phoenix arriostradas (eran iguales en los puentes anteriores que tenian apoyos) en pilastras de mamposteria, que facilitaban el paso de los trenes con las máximas garantías en las épocas de fuertes Iluvias y de riadas. Cumplió perfectamente su cometido y pudo seguir prestando un servicio eficaz una vez cesó la explotación 


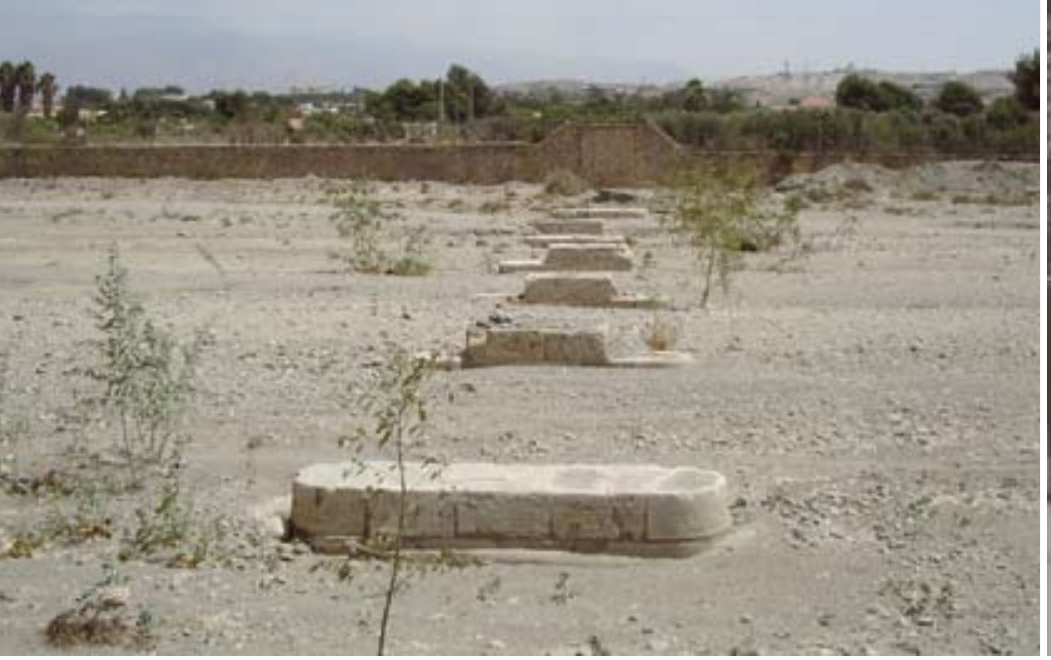

Vestigios del puente del río Andarax. Pilastras de apoyo y estribos de mampostería. Fuente: Fondo ASAFAL

del ferrocarril. En 1942, el Ayuntamiento de Almería obtuvo el permiso para recuperar los tramos metálicos de este puente y de otros para construir dos pasarelas peatonales y un puente carretero sobre la rambla de Belén en la capital almeriense, con lo que conseguian un aprovechamiento total de los materiales en una época muy difícil y de escasez. Con motivo de la reurbanización de esta rambla, a comienzos de la década de los noventa del siglo XX, fue desmontado el puente de la calle Santos Zárate - Paco Aquino (antes habian sido retiradas las pasarelas peatonales de la rambla de Obispo Orberá y de la calle Alcalde Muñoz) y con ello terminó una vida centenaria, primero ferroviaria y después urbana.

Aqui finaliza el recorrido, cuando atraviesa el río Andarax para dirigirse por su margen derecha hasta Almería, pasando por Huércal de Almeria, de cuya estación no queda nada, y discurrir paralelo a la actual linea del ferrocarril de Linares a Almería. Toda esta zona está muy alterada, bien sea por explotaciones agrarias o por viviendas, aunque es de suponer que algunos de los caminos que surcan estos parajes formen parte del antiguo trazado ferroviario, como sucede con la ya mencionada carretera de Sierra Alhamilla de la capital.

Hemos perdido una parte importante del trazado original, pero todavía queda el recorrido más agreste y espectacular para su disfrute. Quizás no esté en las mejores condiciones, pero es posible que, si lo recorremos, conocemos su historia y la divulgamos, seamos capaces de recuperar lo que un día fue sinónimo de progreso.

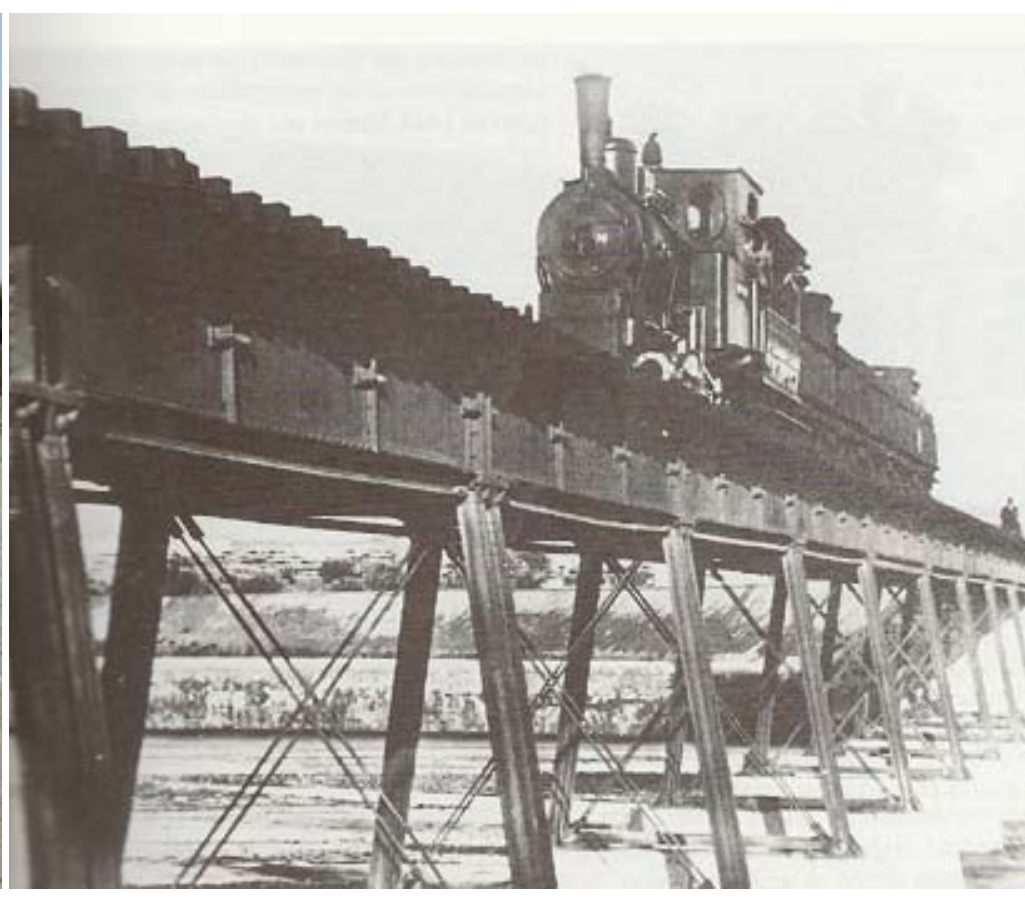

Paso de un tren por el puente del río Andarax. Fuente: Instituto de Estudios Almerienses

\section{Su existencia fue azarosa desde su misma gestación y tuvo una vida corta, sin embargo, nos ha quedado un legado patrimonial que deberíamos proteger del deterioro físico y del olvido}

